\title{
Evaluating the Spatial Dimension of Contacts in Opportunistic Networks
}

[Extended Abstract]

\author{
Nadjet Belblidia \\ UPMC Paris Universitas \\ 104 av. du President Kennedy \\ 75016 Paris, France \\ nadjet.belblidia@lip6.fr
}

\begin{abstract}
Is temporal dimension knowledge alone sufficient to characterize contacts in opportunistic networks? Several studies analyze the temporal aspect of contacts with significant results concerning the contact and inter-contact duration. Nevertheless, only the temporal dimension does not give a complete overview of contact characterization. We propose the surround indicator as a metric to exhibit the contact connectivity in opportunistic networks. We evaluate the surround indicator on two existing datasets. Our preliminary results reveal that contacts have too heterogeneous surrounds to be considered only in terms of duration.
\end{abstract}

\section{Categories and Subject Descriptors}

C.2.1 [Computer-Communications Networks]: Network Architecture and Design-Store and forward networks

\section{General Terms}

Measurement, Experimentation

\section{Keywords}

Delay tolerant networks, capacity, interference.

\section{CONTEXT AND PROBLEM DEFINITION}

One challenging issue in opportunistic networks concerns data transmission optimization. To increase the data delivery probability, it is fundamental to characterize the contact capacity. Providing fine contact capacity characterization is difficult because it depends on a number of parameters that are related to the technology used, the signal propagation laws of the environment, and the traffic demands between source-destination pairs. It is important, however, to take into account any available knowledge to characterize a contact capacity.

Permission to make digital or hard copies of all or part of this work for personal or classroom use is granted without fee provided that copies are not made or distributed for profit or commercial advantage and that copies bear this notice and the full citation on the first page. To copy otherwise, to republish, to post on servers or to redistribute to lists, requires prior specific permission and/or a fee.

MobiOpp '10, February 22-23, 2010, Pisa, Italy.

Copyright 2010 ACM 978-1-60558-925-1/10/02 _...\$10.00.

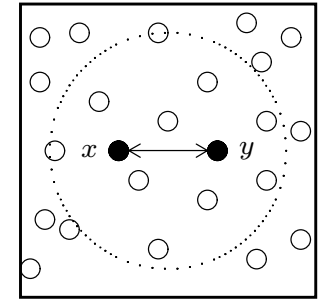

(a) Contact in a dense zone.

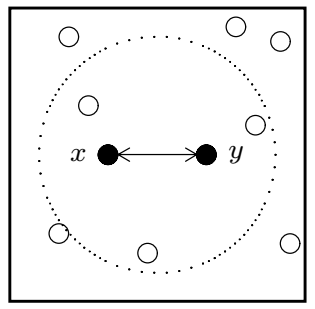

(b) Contact in a sparse zone.
Figure 1: Two situations. The same duration.

A contact has two dimensions: temporal and spatial. The temporal dimension concerns the contact and intercontact distributions and has been the subject of several studies [1, $2,4]$. The spatial dimension is related to the contact's surrounding environment. This dimension can have a real impact on the contact capacity and has been surprisingly underexplored in the literature. Fig. 1 illustrates two completely different situations where contacts last for the same duration. Contacts can be considered as having the same capacity when only considering the temporal dimension. Clearly, although the contacts have the same duration, the one in Fig. 1(a) is much more prone to interference as it happens in a denser zone. We argue that additional information which provides simple indication on the contact surrounding conditions can bring a complementary knowledge for a better contact capacity characterization.

We propose to exhibit the spatial dimension of contacts with information about nearby contacts that are potential sources of interference with the contact under consideration. We define the notion of surround indicator and evaluate its distribution using two datasets with different inherent contact properties. Our preliminary analysis reveals that opportunistic networking is much more dynamic than it is traditionally handled as. Besides the intermittent characteristic of connectivity, nodes observe huge surround variability.

\section{SPATIAL DIMENSION OF CONTACTS}

Let $\mathbf{V}$ be the set of nodes in the network and $\overrightarrow{x y}$ be the directional contact between $x$ and $y$, with $x, y \in \mathbf{V}$. We refer to it as the target contact. $N_{x}^{+}(t)$ and $N_{x}^{-}(t)$ are respectively the sets of out-neighbors and in-neighbors of node $x$ at time $t$. Loosely speaking, we define the surround indicator $\mathcal{S}_{x y}(t)$ 


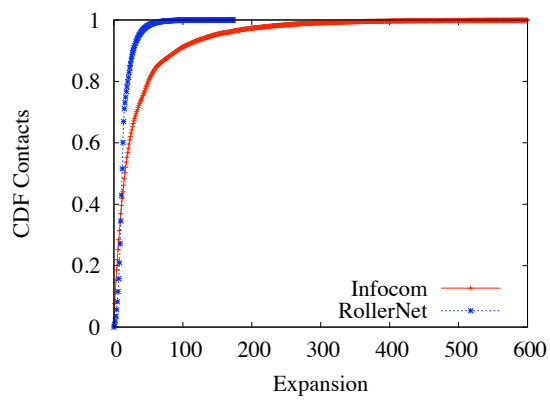

Figure 2: Repartition of contacts according to the expansion.

of the target link $\overrightarrow{x y}$ at time $t$ as the number of outgoing links of all $y$ 's in-neighbors except $x$ :

$$
\mathcal{S}_{x y}(t)=\sum_{\forall z \in N_{y}^{-}(t)-\{x\}}\left|N_{z}^{+}(t)\right| .
$$

From the notation above, it becomes clear that the surround indicator points out how many sources of interference (i.e., collocated contacts) might have an influence on the reception quality of the target contact.

The surround indicator is likely to vary during the interval $\left[t_{1}, t_{2}\right]$, as some neighbors appear while others leave during the contact. We propose to decompose the contact into as many necessary sub-contacts with a stable surround indicator. Each sub-contact represents the temporal view of a surround. We define the expansion as the number of sub-intervals associated to the same original contact.

This representation allows not only characterizing neighborhood density through the surround indicator, but also to have a clue on the network dynamics. Indeed, the expansion reflects the contact surround instability.

\section{PRELIMINARY EVALUATION}

We evaluate the spatial dimension of contacts in the Infocom [1] and RollerNet [5] datasets. These datasets are publicly available to the community through the Crawdad repository [3].

Fig. 2 shows the distribution of the contacts according to the expansion value. In RollerNet, about $50 \%$ of the contacts have an expansion larger than 12. Similarly, in Infocom, about $50 \%$ of the contacts have an expansion larger than 16. Furthermore, the expansion reaches extreme values in both cases. In RollerNet, the most instable surround contact has an expansion equal to 173 , while it reaches 1,436 in Infocom.

Fig. 3 shows the cumulative distribution function of subcontacts according to the surround indicator. For both datasets, the percentage of contacts with a null surround indicator does not exceed $0.55 \%$ for RollerNet and $0.59 \%$ for Infocom. Therefore, it is very rare that a contact happens in an isolated environment. Moreover, in extreme cases, the surround indicator reaches 212 in RollerNet and 378 in Infocom. Even if the surround indicator is just an indication of potential parallel communications, such a high number of surrounding contacts clearly shows that some zones are likely to have a limited number of working links, if any, due to mutual interference (if only because of exchanged control messages).

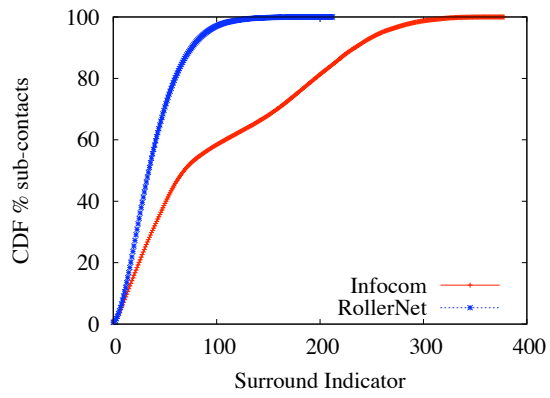

Figure 3: Repartition of sub-contacts according to the surround indicator.

\section{CONCLUSION AND FUTURE WORK}

In this abstract, we introduce the surround indicator as a metric to exhibit the spatial dimension of contacts in opportunistic networks. The surround indicator provides explicit information of the environment in which contact takes place. Our preliminary analyse on two different datasets shows that the surrounding environment of a contacts is more dynamics than indicated by solely contact and intercontact times.

Although the proposed metric is merely indicative, the surround indicator can be useful in a number of research problems. As future work, a routing protocol might decide to avoid a path that traverses a node that has a too high surround indicator value (thus prone to competitive flows). Similarly, the same node might be a good starting point of an epidemic dissemination algorithm.

\section{ACKNOWLEDGMENTS}

This work is being done under the supervision of my advisors Marcelo Dias de Amorim and Serge Fdida from UPMC Paris Universitas in collaboration with Jérémie Leguay and Vania Conan from Thalès Communications and Jon Crowcroft from Cambridge University. This work is partially supported by the ANR project Crowd under contract ANR08-VERS-006.

\section{REFERENCES}

[1] A. Chaintreau, P. Hui, C. Diot, R. Gass, and J. Scott. Impact of human mobility on opportunistic forwarding algorithms. IEEE Transactions on Mobile Computing, 2007.

[2] V. Conan, J. Leguay, and T. Friedman. Characterizing pairwise inter-contact patterns in delay tolerant networks. In International conference on Autonomic computing and communication systems, ICST, Brussels, Belgium, 2007.

[3] CRAWDAD. http://crawdad.cs.dartmouth.edu.

[4] T. Karagiannis, J.-Y. L. Boudec, and M. Vojnović. Power law and exponential decay of inter contact times between mobile devices. In ACM Mobicom, New York, NY, USA, 2007. ACM.

[5] P. U. Tournoux, J. Leguay, F. Benbadis, V. Conan, M. D. de Amorim, and J. Whitbeck. The accordion phenomenon: Analysis, characterization, and impact on dtn routing. In IEEE Infocom, 2009. 